\title{
Nonstoichiometry, Defects and Transport Properties in $\mathrm{MgB}_{2}$
}

\author{
Y. Y. Xue, R. L. Meng, B. Lorenz, J. K. Meen, Y. Y. Sun and C. W. Chu* \\ TCSUH, University of Houston, Houston, TX 77204-5932 ('also at \\ LBNL, 1 Cyclotron Road, Berkeley, CA 94720)
}

(November 15, 2018)

Typeset using REVTEX 


\begin{abstract}
The local composition $\mathrm{Mg}: \mathrm{B}$ of $\mathrm{MgB}_{2}$ powder was systematically changed through annealing. Correlations were observed between the $\mathrm{Mg}$ loss and the lattice parameters $a / c$ and the microstrain of $\mathrm{MgB}_{2}$. Direct wavelengthdispersive-spectrum and XRD measurements on ceramic samples with different residual resistivity ratios suggested that the transport property of $\mathrm{MgB}_{2}$ might be affected by this Mg-deficiency, but oxygen-related defects may play a more important role.
\end{abstract}

One unsolved issue in the transport property of the newly discovered superconductive $\mathrm{MgB}_{2}$ is its apparent sample-dependency. The reported resistivity at room-temperature, $\rho(297 \mathrm{~K})$, ranges from 9.6 to $100 \mu \Omega \mathrm{cm}$, and that at $40 \mathrm{~K}, \rho(40 \mathrm{~K})$, from 0.38 to $21 \mu \Omega \mathrm{cm}$. 1. . Both the $\rho(297 \mathrm{~K})$ and the residual resistivity ratio, $\mathrm{RRR}=\rho(297 \mathrm{~K}) / \rho(40 \mathrm{~K})$, vary by a factor of ten among ceramic samples purported to be high-quality. In a closely related way, the reported magnetoresistance, $\Delta \rho / \rho=[\rho(40 \mathrm{~K}, 5 \mathrm{~T})-\rho(40 \mathrm{~K}, 0 \mathrm{~T})] / \rho(40 \mathrm{~K}, 0 \mathrm{~T})$, of ceramic $\mathrm{MgB}_{2}$ samples varies from $<1 \%$ to $60 \%$ (i.e. more than 100 times), 3 and the temperature dependency of the resistivity varies from $\mathrm{T}^{2}$ to $\mathrm{T}^{3}$. may depend on its porosity and grain coupling, the large variations in RRR and $\Delta \rho / \rho$ may require dense lattice defects. Resistances of grain-boundaries typically will only add a constant in $\rho$, which should lead to a linear correlation of $\Delta \rho / \rho \propto 1 / \operatorname{RRR}$ instead of the $\Delta \rho / \rho$ $\propto(1 / \mathrm{RRR})^{2}$ observed 5 The sample dependency has been previously attributed to either the pressure or the thermal history used during the synthesis. 6 However, the fact that similar low RRR and negligible magnetoresistance have been observed in samples synthesized both with and without high-pressures challenges the interpretation. The mechanism responsible for the differences needs to be explored.

It is interesting to note that the local composition, i.e. both the atomic ratio $\mathrm{B}: \mathrm{Mg}$ and the possible oxygen contamination, is still a topic of debate. Cooper et al. proposed in 1970 that the boron in borides $\mathrm{AB}_{2}$ might be nonstoichiometric, 0 and Zhao et al. reported that the superconducting temperature, $\mathrm{T}_{C}$, of $\mathrm{MgB}_{2}$ changes significantly with different starting compositions of $\mathrm{Mg}: \mathrm{B} .8$ Most $\mathrm{MgB}_{2}$ samples, it should be pointed out, were synthesized with a stoichiometric starting composition. Noticeable amounts of $\mathrm{MgO}$ and/or $\mathrm{Mg}$ are either frozen inside the samples or deposited on the walls of the sample containers. $\mathrm{MgB}_{2}$ samples, therefore, could be non stoichiometric. The facts that Au foil, which is typically used in high-pressure synthesis, can easily absorb $\mathrm{Mg}$, and the samples with large RRR were synthesized using excess $\mathrm{Mg}, \mathrm{D}$ may further enhance the suspicion. On the other hand, the EELS data of Zhu et al. shown that the local $\mathrm{Mg}: \mathrm{B}$ is stoichiometric in their samples with a variation less than $\pm 2 \%$ The $\mathrm{Mg}$-stoichiometry and its effect on transport property, therefore, may still be open questions.

Oxygen contamination has also been widely discussed, in particular in terms of the $\mathrm{T}_{C}$ degrading of $\mathrm{MgB}_{2}$ films. 10 However, the $\mathrm{T}_{C}$ of a ceramic with a grain size of $1 \mu \mathrm{m}$ or larger typically will not be affected unless the contamination causes lattice defects and/or microstrain. Zhu et al. have shown that $\mathrm{MgO}$ can exist inside $\mathrm{MgB}_{2}$ grains as stacking faults and create microstrains 9 The effects of these faults, consequentially, will depend on their morphology (therefore, oxygen concentration, diffusion and synthesis temperatures). It should be cautioned, that the Mg powder/chips used may also function as an oxygen 
getter, and the oxygen related defects may also be affected by the starting composition.

To explore the topic, we measured X-ray diffraction (XRD), composition and resistivity of several $\mathrm{MgB}_{2}$ ceramic samples. A systematic correlation among RRR, the lattice distortion (microstrain) and intragrain oxygen-concentration was observed. The local ratio of $\mathrm{Mg}: \mathrm{B}$ of two ceramic $\mathrm{MgB}_{2}$ samples with rather different $\mathrm{RRR}$, however, is almost the same. These suggest that the transport property of $\mathrm{MgB}_{2}$ is dominated by the oxygen related defects. It should be pointed out, however, a $\leq 5 \% \mathrm{Mg}$ off stoichiometry was observed in $\mathrm{MgB}_{2}$ powders annealed at low temperatures. An accompanied systematic change of the lattice parameters/microstrain further suggested that this off stoichiometry may also create lattice defects, although the fast diffusion of $\mathrm{Mg} / \mathrm{B}$ may make such defects disappear and play only minor roles in typical $\mathrm{MgB}_{2}$ ceramic.

Ceramic $\mathrm{MgB}_{2}$ samples were prepared using the solid-state reaction method. 3 A mixture of small Mg chips (99.8\% pure) and B powder (99.7\% pure) were sealed inside a Ta tube under an Ar atmosphere. The sealed Ta ampoule was then enclosed in a quartz tube. The assembly was heated slowly up to $950{ }^{\circ} \mathrm{C}$ followed by rapid quenching. Various starting $\mathrm{Mg}: \mathrm{B}$ ratios were used to obtain samples with different RRR's. Unfortunately, the impurity phases in the samples so prepared make a quantitative $\mathrm{Mg}$ stoichiometry-control difficult. Several Mg-deficient samples, therefore, were made by vacuum annealing commercial $\mathrm{MgB}_{2}$ powder (Alfa, 98\% pure). The structure was determined by X-ray powder diffraction (XRD) using a Rigaku DMAX-IIIB diffractometer with a $\mathrm{Cu}$ target. The refinement was done using the Reitan-94 program.11 The resistivity was measured through the standard 4-probe method using a R-700 ac bridge. Analysis of the magnesium boride grains was done by electron microprobe analysis (EMPA) at an accelerating voltage of $15 \mathrm{kV}$ and $30 \mathrm{nA}$ beam current using a JEOL JXA 8600 electron microprobe with attached Wavelength Dispersive Spectrometers (WDS).

To answer the question of whether $\mathrm{MgB}_{2}$ can be microscopically non-stoichiometric, commercial Alfa $\mathrm{MgB}_{2}$ powder was annealed in pre-vacuumed quartz tubes at various temperatures between 700 and $900{ }^{\circ} \mathrm{C}$ followed by rapid quenching. The weight loss during the annealing was measured. X-ray powder diffraction was then performed and the data refined as a mixture of $\mathrm{MgB}_{2}$ (space group I-191) and $\mathrm{MgO}$ (I-225) with only the line-profile parameters, the phase composition $\mathrm{MgO} / \mathrm{MgB}_{2}$, the lattice constants of $\mathrm{MgB}_{2}$, and the isotropic thermal factor adjustable. The lines of impurity phases other than $\mathrm{MgO}$ were not used during the refinement. A typical data set is shown in Fig. 1. Typically, the R-pattern, $R_{p}$, the goodness-of-fit, $S$, and the R-structure factor of $\mathrm{MgB}_{2}, R_{F}$, are 4, 1.4, and 2, respectively, an indication of reasonable agreement.12 It was noted, however, that the spread of the refined parameters was larger than the mathematical uncertainty $\sigma$ given by the program. The lattice constants, for example, can spread over $\pm 0.0005 \AA$ from one sample to another with the same $\mathrm{Mg}$ deficiency while the $\sigma$ is only \pm 1 to $2 \cdot 10^{-4} \AA$. To estimate the experimental resolution, therefore, the samples with the same weight loss were prepared and measured repeatedly. The data spread was used as the experimental resolution in following figures. During the annealing, the fraction of $\mathrm{MgO}$ seemed to increase from $8 \%$ of the Alfa powder to $\approx 14 \%$ of the annealed samples. The weight loss and the ratio $\mathrm{MgO} / \mathrm{MgB}_{2}$, therefore, were combined to calculate the change of the $\mathrm{Mg}$ composition in $\mathrm{MgB}_{2}$, i.e. the $1-\mathrm{x}$ in $\mathrm{Mg}_{1-x-x o} \mathrm{~B}_{2}$, assuming that the Alfa powder is $\mathrm{Mg}_{1-x o} \mathrm{~B}_{2}$. Other impurity phases, such as $\mathrm{MgB}_{4}$, appear when $1-\mathrm{x}<0.86$ and this estimation can no longer be used. It should be noted 
that it might also be possible that the Mg deficiency caused by the formation of $\mathrm{MgO}$ might be spatially very inhomogeneous, and the 1-x assumed could have a systematic shift as large as 0.06 .

A noticeable trend was observed in a decrease of the lattice parameter $c$, and an increase of $a$ with increasing of 1-x (Fig. 2). To verify that this change is not due to some unknown effect of the heat treatment, metal Mg chips were added to one of the quartz tubes to prevent Mg-loss during the annealing. The weight loss and the change in the $\mathrm{MgO}$ fraction observed were indeed the same as those of the initial Alfa powder. The $a$ and $c$ of this Mg-added sample (the triangles in Fig. 2) are identical to those of the Alfa powder, demonstrating that Mg-loss during the anneal is the main cause of the change in lattice parameters. This systematic change of $a / c$ will not be possible without a noticeable off-stoichiometry of Mg, which is different from the almost ideal composition observed by Zhu et al, 9 although the missing or intercalation of boron/magnesium planes observed there show that an off-stoichiometry should be possible in principle. We attribute it to the differences in sample-preparation, e.g. the lower annealing temperature used here may freeze the $\mathrm{Mg}$ - and B-related defects. It should be noted that no significant variation in $a / c$ was observed with $1-\mathrm{x}<0.85$, suggesting a phase-stability boundary around $\mathrm{Mg}: \mathrm{B} \approx 0.85: 2$. Variation of $a / c$ was reported previously with a much broader $\mathrm{Mg}: \mathrm{B}$ range (0.5:2 to 2:2) Significant impurity phases, e.g. $\mathrm{MgB}_{4}$, in the samples may cause the difference based on the reported XRD.

A more subtle but important change associated with the Mg-loss is the broadening of the XRD lines. The line width significantly increases with the loss of Mg (Fig. 3), although it is already much larger than our instrumental resolutions in the initial Alfa powder. It should be noted that the true change in the line profile would be much more prompt if there were no interference between the $\mathrm{Cu} \mathrm{K}_{\alpha 1}$ and $\mathrm{K}_{\alpha 2}$ lines. The significant contribution from the $\mathrm{K}_{\alpha 2}$ line, unfortunately, makes a detailed analysis of the anisotropic strain impractical. We, therefore, have to depend on the Rietveld refinement. An isotropic broadening was assumed. i.e. the line profile was fitted with the pseudo-Voigt function of the scattering angle $2 \theta .12$ Mathematically, the function is a convolution of a Lorentz function with a Gauss function. To deduce the instability of the nonlinear regression, the variance $\sigma^{2}$ of the Gauss function was taken as $U \tan ^{2} \theta+V \tan \theta+W$, i.e. ignoring the Scherrer term $P \sec ^{2} \theta$ since it is rather small anyway. The parameters $\mathrm{V}$ and $\mathrm{W}$, which are not expected to be sample-dependent, were further fixed to the values obtained in a calibration run with well-crystallized $\mathrm{Al}_{2} \mathrm{O}_{3}$ powder. Similarly, the full-width-at-half-maximum (FWHM) of the Lorentz function $\mathrm{H}_{k L}$ was assumed to be $X \cdot \sec \theta+Y \cdot \tan \theta$, i.e. ignoring the anisotropic broadening terms $X_{e}$ and $Y_{e} .21$ Previously, the parameters $U$ and $Y$, i.e. the components varying as $\tan \theta$, were used to characterize the microstrain, i.e. either a modulation of local lattice parameters or their domain-to-domain variation. The $\sec \theta \operatorname{component} X$, on the other hand, was generally used to describe the size of the structural domains.

The $U$ and $Y$ of the annealed powders are shown in Fig. 4. While $U$ seems to increase with $\mathrm{x}$ linearly over the whole range probed, $Y$ seems to be noticeably affected only beyond a threshold $\mathrm{Mg}$ loss of $\mathrm{x} \approx 0.1$. The line profile seems to be dominated by the Gauss components in the slightly Mg-deficient samples, but by the Lorentz components in the heavily $\mathrm{Mg}$-deficient ones. The value of $X$, on the other hand, shows no systematic change with $\mathrm{x}$. The widening of the XRD lines, therefore, is mainly related to the local variation of the lattice parameters. We further attribute it to the non-stoichiometry of $\mathrm{MgB}_{2}$ based on 
the systematic changes of the $a$ and $c$ with the x observed. To qualitatively estimate this microstrain, the overall FWHM of the pseudo-Voigt functions with the $U$ and $Y$ equal to the observed values was calculated as $\mathrm{H}_{k}$, and that with $U=Y=0$ as $\mathrm{H}_{k 0}$. The contribution of microstrain was then taken as $\Delta \theta=\sqrt{\mathrm{H}_{\mathrm{k}}^{2}-\mathrm{H}_{\mathrm{k} 0}^{2}}$. The weighted relative variation of the $a$ and $c$ (the microstrain), therefore, should be of the order of $\varepsilon=\Delta \theta / \tan \theta$. The microstrain socalculated is almost $\theta$-independent between $\theta=10^{\circ}$ and $40^{\circ}$ in a given sample, as expected. A systematic increase of $\varepsilon$ with the loss of $\mathrm{Mg}$ was observed (Fig. 5). It seems that the Mg-loss is extremely non-uniform, and the resulting local defects could serve as scattering centers for the carriers.

Despite their differences in composition and lattice parameters, however, the superconductivity measured on these samples were almost the same (Fig. 6). The onset of the zero-field-cooled magnetization, $\mathrm{M}_{Z F C}$, is $39 \mathrm{~K}$ and the transition width $(10 \%$ to $80 \%$ of $\mathrm{M}_{Z F C}$ at $5 \mathrm{~K}$ ) differs by less than $0.5 \mathrm{~K}$ from sample to sample. This may be understood in terms of percolation and the fact that $\mathrm{MgB}_{2}$, as a S-wave BCS superconductor, should not be sensitive to defects. The observation that $\mathrm{Mg}$ nonstoichiometry alone will not cause large $\mathrm{T}_{C}$ change is also supported by our data on several samples synthesized with starting composition $\mathrm{Mg}: \mathrm{B}$ from 0.5:2 to 2.4:2.14 It is interesting to note that the field-cooled magnetizations, $\mathrm{M}_{F C}$, were also sample-independent. The similar vortex pinning strength suggests that the length scale between defects might be comparable or smaller than the coherent length $\left(\approx 60 \AA\right.$ for $\left.\mathrm{MgB}_{2}\right)$.

Unfortunately, no transport measurement can be made in these annealed samples, which were made in the powder form to ensure the uniform loss of $\mathrm{Mg}$. Therefore, additional ceramic samples were purposely made with different starting compositions. These samples show different RRR. The $\rho(\mathrm{T})$ of two typical samples with $\mathrm{RRR}=2.5$ (Sample A) and 7 (Sample B), respectively, are shown in Fig. 7. Sample B was synthesized with extra Mgchips and a start composition of $\mathrm{Mg}: \mathrm{B}=1.25: 2$. It is expected, therefore, that Sample $\mathrm{B}$ should be relatively $\mathrm{Mg}$-rich and $\mathrm{O}$-free.

The $c(a)$ is 3.525 and $3.519 \AA$ (3.084 and $3.085 \AA$ ) observed for Sample A and B, respectively. While the shorter $c$ of Sample B seems to be in qualitative agreement with the assumption that it is Mg-rich, the similar a observed suggests that other defects may also play a role. In fact, the estimated 1-x of Sample B would be 1.2 based on its $c$, but 1.03 based on the $a$ using Fig. 2 as the calibration curve. To further explore the topic, $a / c$ of several ceramic samples with RRR from 2.3 to 7 were measured (Fig. 8). When the $c$ appears to decrease systematically with RRR, no clear correlation exists between the $a$ and RRR. Defects other than Mg-deficiency seem to play a role, based on a comparison between Figs. 2 and 8.

To verify the $\mathrm{Mg}$ stoichiometry in Samples A and B, the samples were subjected to EMPA analysis for $\mathrm{Mg}, \mathrm{B}, \mathrm{Si}$, and $\mathrm{O}$, together with a cool-pressed Alfa $\mathrm{MgB}_{2}$ powder. The observed ratio $\mathrm{B}: \mathrm{Mg} \approx 2.01: 1$ for the Alfa powder sample has a standard deviation of $\pm 0.04(\approx 2 \%)$ over 12 positions measured. Similarly, the ratio is 1.99 $\pm 0.03: 1$ for Sample A, but 2.06 $\pm 0.17: 1$ for Sample B. The greater variability of the composition of Sample B is plausibly the result of its much smaller grain size $(<2 \mu \mathrm{m}$ in diameter $)$. The regions in the grain boundaries introduces an additional complicating factor. It is interesting to note, however, that the point-to-point variation in Sample A and the Alfa powder still exceeds the precision of our analysis $(\approx 0.4 \%)$, although their grains are much larger. This may be 
an indication of a real composition variability, and in agreement with the nonstoichiometry discussed above. In such a case, the data after the average should be much closer to the true value. The almost identical average compositions of $1-\mathrm{x} \approx 1.01$ and 0.97 in Samples $\mathrm{A}$ and $\mathrm{B}$, respectively, show that the nonstoichiometry may not be the dominating factor for the different RRR observed in ceramic samples. It is interesting to note that the $\mathrm{Mg}$ off-stoichiometry of these as-synthesized $\mathrm{MgB}_{2}$ ceramic samples is rather small, which is in agreement with the EELS data of Zhu et al.

However, a clear trend was observed between RRR and the microstrain $\varepsilon$. The raw XRD data already show that the line width of Sample B is significantly narrower (Fig. 3). The refinement further demonstrate that most of the narrowing comes from the decrease of $U$ and $Y$ (the microstrain terms). In fact, the combined $\varepsilon$ is $0.004,0.01$, and 0.008 for Samples A and $\mathrm{B}$ and the Alfa powder, respectively. To verify the assumption, the $\varepsilon$ of several ceramic samples with RRR from 2.2 to 7 was also measured (Fig 9). The $\varepsilon$ decreases with RRR, and a strain $<0.2 \%$ seems to be needed for $R R R \approx 20$. The residual resistance, therefore, seems to be related to a lattice distortion other than $\mathrm{Mg}$ nonstoichiometry.

Indeed, a correlation was observed between the RRR and local oxygen composition in several ceramic $\mathrm{MgB}_{2}$ samples. The atomic ratio $\mathrm{O} / \mathrm{Mg}$, for example, was $0.15 \pm 0.03$ and $0.08 \pm 0.03$ for Samples A and B, respectively. It should be pointed out that the extra oxygen observed in Sample A is unlikely to come from separated $\mathrm{MgO}$ grains based on the observed grain size $(1-5 \mu \mathrm{m})$ and the estimated space resolution (see appendix). It is also difficult to attribute the contamination to surface oxide layers. Sample B, for example, has much smaller grain size and should have severe oxygen contamination if the oxygen is mainly from grain surfaces. We, therefore, suggest that oxgen may exist inside $\mathrm{MgB}_{2}$ grains as defects, which is in agreement with the $\mathrm{MgO} / \mathrm{MgB}_{2}$ stacking faults observed by Zhu et

al. 9 To verify that, the ratio O:Mg in other five samples were measured (Fig. 10). The trend is clear, although the data scattering is large.

In summary, our data suggest that $\mathrm{MgB}_{2}$ can be nonstoichiometric up to $5-10 \%$. Although this nonstoichiometry may affect the transport properties, oxygen related defects seem to play dominating roles in $\mathrm{MgB}_{2}$.

ACKNOWLEDGMENT This work is supported in part by NSF Grant, the T. L. L. Temple Foundation, the John and Rebecca Moores Endowment and the State of Texas through TCSUH, and in LBNL by DOE.

\section{APPENDIX}

Analysis of the magnesium boride grains was done by electron microprobe analysis (EMPA) using an accelerating voltage of $15 \mathrm{kV}$ and $30 \mathrm{nA}$ beam current. Samples were set in epoxy and polished to $1 \mu \mathrm{m}$ relief. Primary standards used were $\mathrm{MgO}$ for $\mathrm{Mg}$ and $\mathrm{O}$, but pure boron for B. Peaks were counted for $100 \mathrm{~s}$ and two backgrounds for $50 \mathrm{~s}$ each for each element. A $20 \mu \mathrm{m}$ spot size was used for standards but a focused beam was used on the sample grains. Calculations based on electron-target atom interactions suggest that $>99 \%$ of characteristic $\mathrm{x}$-rays for samples are derived from a region with a $\approx 2.5 \mu \mathrm{m}$ diameter. Characteristic x-rays derived from boron atoms with an energy of $\approx 183 \mathrm{eV}$ are derived from the top $1.4 \mu \mathrm{m}$ of the sample. Those of $\mathrm{Mg}(1253 \mathrm{eV})$ are from the top $1.7 \mu \mathrm{m}$. Oxygen $\mathrm{x}$-ray is from the top $2.0 \mu \mathrm{m}$. Thus, the volumes sampled by the x-rays are similar for each element and are $\approx 10 \mu \mathrm{m}^{3}$.

Characteristic x-rays of each element were separated from other photons using 
wavelength-dispersive spectrometers employing a crystal of thallium acid phthalate $(\mathrm{Mg})$ and synthetic layered dispersive elements (B, O). The energy of the Mg K $\alpha$ x-rays are identical within reproducibility for standards and samples. The characteristic x-rays of both $\mathrm{B}$ and $\mathrm{O}$ do, however, indicate significant changes in energy between samples and standards and even between different orientations of the magnesium boride crystalline samples. This reflects differences in the energy levels occupied by the $2 p$ electrons in these atoms. The spectrometer position required to maximize the characteristic $\mathrm{B} \mathrm{K} \alpha$ x-ray was, therefore,

determined for each sample before each analysis. Careful determination of the FWHM of the x-ray peak indicated that the shape of the peak (as opposed to the mean energy) did not vary significantly from the pure B standard to the samples nor between the different samples.

The raw x-ray intensities were corrected using the on-line Geller iterative procedure. The resultant corrections were very large for $\mathrm{B}$, in particular. Thus, the corrected fraction of boron in a sample was computed to be approximately ten times the fraction of boron estimated by comparing raw intensities of x-rays on sample and standard. The corrected fraction of $\mathrm{Mg}$ is typically 0.5 the raw estimate. These corrections reflect the large differences in inter-element correction factors between standards and samples. Commercial $\mathrm{MgB}_{2}$ was therefore used as a secondary standard for all analyses. The secondary standard was carboncoated at the same time as the samples in each case. Routine techniques allow control of the thickness of the carbon coat to within $\approx 10 \%$ of the nominal $200 \mathrm{~nm}$. The marked absorption of characteristic B x-rays by carbon warrants an even higher level of control and this was monitored by determining the intensity of boron x-rays emitted from the secondary standard. 


\section{REFERENCES}

${ }^{1}$ P. C. Canfield, D. K. Finnemore, S. L. Bud'ko, J. E. Ostenson, G. Lapertot, C. E. Cunningham and C. Petrovic, cond-mat/0102289

${ }^{2}$ C. U. Jung, M. S. Park, W. N. Kang, M. S. Kim, S. Y. Lee and S. I. Lee, condmat/0102215.

${ }^{3}$ S. L. Bud'ko, C. Petrovic, G. Lapertot, C. E. Cunningham, P. C. Canfield, M. H. Jung, A. H. Lacerda, cond-mat/0102413.

${ }^{4}$ W. N. Kang, C. U. Jung, K. H. P. Kim, M. S. Park, S. Y. Lee, H. J. Kim, E. M. Choi, K. H. Kim, M. S. Kim and S. I. Lee, cond-mat/0102313.

${ }^{5}$ X. H. Chen, Y. S. Wang, Y. Y. Xue, R. L. Meng, Y. Q. Wang and C. W. Chu, unpublished

${ }^{6}$ C. E. Cunningham, C. Petrovic, G. Lapertot, S. L. Bud'ko, F. Laabs, W. Straszheim, D. K. Finnemore and P. C. Canfield, cond-mat/0103390.

${ }^{7}$ A. S. Cooper, E. Corenzwit, L. D. Longinotti, B. T. Matthias and W. H. Zachariasen, Proc. Natl. Acad. Sci. 67, 313 (1970).

${ }^{8}$ Y. G. Zhao, X. P. Zhang, P. T. Qiao, H. T. Zhang, S. L. Jia, B. S. Cao M. H. Zhu, Z. H. Han, X. L. Wang and B. L. Gu, cond-mat/0105053.

${ }^{9}$ Y. Zhu, L. Wu, V. Volkov, Q. Li, G. Gu, A. R. Moodenbaugh, M. Malac, M. Suenaga and J. Tranquada, cond-mat/0105311.

${ }^{10}$ H. Y. Zhai, H. M. Christen, L. Zhang, M. Parathaman, C. Cantoni, B. C. Sales, P. H. Fleming, D. K. Christen and D. M. Lowndes, cond-mat/0103618.

${ }^{11}$ F. Izumi, Rigaku J. 6, 10 (1989).

${ }^{12}$ For example, "The Rietveld Method", edited by R. A. Young (New York, Oxford University Press, 1993).

${ }^{13}$ The value of $P$ is rather small when set as free parameters in some test runs, and not expected to affect our conclusion significantly.

${ }^{14} \mathrm{R}$. L. Meng et al unpublished. 


\section{FIGURES}

FIG. 1. The XRD pattern of the initial $\mathrm{MgB}_{2}$ powder (98\%, Alfa). $\because$ data; top solid line: Rietveld refinement; bottom solid line: residual; \#: lines of $\mathrm{MgO}$; *: lines of unknown impurity.

FIG. 2. The lattice parameters $a$ (open symbols) and $c$ (filled symbols). $\nabla, \boldsymbol{\nabla}$ : initial Alfa powder; $\bigcirc$, •: annealed in vacuumed tube; $\square, \mathbf{\square}$ : annealed together with Mg-chips.

FIG. 3. Line-profiles of the 002 line of $\mathrm{MgB}_{2}$ at $51.8^{\circ}$. solid line: the initial Alfa powder; dashed line: an annealed powder; dotted line: a sample with a resistivity ratio $R R R=5$; dotted-dashed line: the 024 line of a $\mathrm{Al}_{2} \mathrm{O}_{3}$ sample at $52.3^{\circ}$ to show the instrumental resolution. The lines have been parallel shifted and normalized to emphasize the line width.

FIG. 4. The profile parameters $U$ (open symbols) and $Y$ (filled symbols). $\nabla$, $\mathbf{v}$ : initial Alfa powder; $\bigcirc$, •: annealed in tube; $\square, \mathbf{\square}$ : annealed together with $\mathrm{Mg}$-chips.

FIG. 5. The estimated isotropic microstrain $\varepsilon$ vs. Mg-loss of 1-x. $\nabla$ : initial Alfa powder; $\bigcirc$ : annealed in tube; $\square$ : annealed together with Mg-chips.

FIG. 6. $\mathrm{M}_{Z F C}$ (open symbols) and $\mathrm{M}_{F C}$ (filled symbols) of two sample with $1-\mathrm{x}=0(\bigcirc, \bullet)$ and $0.85(\triangle, \boldsymbol{\Delta})$, respectively.

FIG. 7. $\rho(\mathrm{T})$ of two typical samples. +: data; solid lines: $\mathrm{a}+\mathrm{bT}^{n}$ fits.

FIG. 8. The lattice parameters $a(\bullet)$ and $c(\boldsymbol{\nabla})$ as functions of RRR.

FIG. 9. The strain $\varepsilon$ vs. RRR

FIG. 10. Oxygen concentration $\mathrm{O} / \mathrm{Mg}$ vs. RRR 
This figure "fig01.png" is available in "png" format from: http://arxiv.org/ps/cond-mat/0105478v1 
This figure "fig02.png" is available in "png" format from: http://arxiv.org/ps/cond-mat/0105478v1 
This figure "fig03.png" is available in "png" format from: http://arxiv.org/ps/cond-mat/0105478v1 
This figure "fig04.png" is available in "png" format from: http://arxiv.org/ps/cond-mat/0105478v1 
This figure "fig05.png" is available in "png" format from: http://arxiv.org/ps/cond-mat/0105478v1 
This figure "fig06.png" is available in "png" format from: http://arxiv.org/ps/cond-mat/0105478v1 
This figure "fig07.png" is available in "png" format from: http://arxiv.org/ps/cond-mat/0105478v1 
This figure "fig08.png" is available in "png" format from: http://arxiv.org/ps/cond-mat/0105478v1 
This figure "fig09.png" is available in "png" format from: http://arxiv.org/ps/cond-mat/0105478v1 
This figure "fig10.png" is available in "png" format from: http://arxiv.org/ps/cond-mat/0105478v1 